\title{
DNA-dependent Protein Kinase Inhibitor VX-984
}

National Cancer Institute

\section{Source}

National Cancer Institute. DNA-dependent Protein Kinase Inhibitor VX-984. NCI

Thesaurus. Code C129061.

An ATP-competitive inhibitor of the catalytic subunit of DNA-dependent protein kinase (DNA-PK), with potential sensitizing and enhancing activities for both chemo- and radiotherapies. Upon administration, DNA-PK inhibitor VX-984 binds to and inhibits the catalytic subunit of DNA-PK, thereby interfering with the non-homologous end joining (NHEJ) process and preventing repair of DNA double strand breaks (DSBs) caused by ionizing radiation or chemotherapeutic treatment. This increases chemo- and radiotherapy cytotoxicity and leads to enhanced tumor cell death. The enhanced ability of tumor cells to repair DSBs plays a major role in the resistance of tumor cells to chemoand radiotherapy; DNA-PK plays a key role in the NHEJ pathway and DSB repair. 